\title{
EDUCACIÓN INTERCULTURAL: REFLEXIONES SOBRE DOS DÉCADAS DE INTERCULTURALISMO EN MÉXICO
}

\author{
EDUCAÇÃO INTERCULTURAL: REFLEXÕES SOBRE DUAS DÉCADAS DE \\ INTERCULTURALISMO NO MÉXICO
}

\section{INTERCULTURAL EDUCATION: REFLECTIONS ON TWO DECADES OF INTERCULTURALISM IN MEXICO}

Nicanor Rebolledo ${ }^{19}$

\begin{abstract}
Resumo
O trabalho reflete sobre as políticas de educação intercultural das últimas duas décadas no México. Inicia com uma apresentação das questões que se debatem neste período e as reflexões que a política intercultural desencadeia. Em seguida, analisa as novas burocracias que foram criadas nessas duas décadas para promover as políticas interculturais, que buscavam não apenas desmontar o antigo dispositivo de integração indígena, mas também estabelecer uma política de livre mercado, "de bocho e changarro", apoiada em reformas que desencadearam mudanças na gestão dos recursos destinados à educação indígena e no jogo aberto na tomada de decisões sobre o tipo de oferta escolar (conteúdos de aprendizagem e avaliação). Para finalizar, faz uma revisão de algumas posições críticas que associam o interculturalismo com o neoliberalismo e que, para escapar desse círculo é urgente desenvolver um discurso crítico e decolonial. Movimentos indígenas que lutam não apenas pelos direitos culturais, educacionais e linguísticos, mas contra o capitalismo global.
\end{abstract}

Palavras-chave: Interculturalismo. Educação intercultural. Neoliberalismo. Indigenismo.

\section{Resumen}

El trabajo reflexiona sobre las políticas de educación intercultural de las últimas dos décadas en México. Inicia con una presentación de los temas que se debaten durante este periodo y las reflexiones que suscita la política intercultural. Enseguida entra a analizar la burocracia de los aparatos que se crearon durante estas dos décadas para impulsar las políticas interculturales, que no solo buscan desmantelar el viejo aparato

\footnotetext{
${ }^{19}$ Doctor en Antropología Social, profesor en la Licenciatura en Educación Indígena, la Maestría en Desarrollo Educativo y en los Doctorados de Educación y Educación y Diversidad de la Universidad Pedagógica Nacional-México. Investigador del Sistema Nacional de Investigadores Nivel 2. Correo: nrebolle@upn.mx, https://orcid.org/0000-0003-0024-7727
} 


\title{
RevistAleph
}

indigenista de integración, sino de instaurar una política de libre mercado, "de bocho y changarro", apoyados en reformas que desencadenaron cambios en el manejo de los recursos destinados a la educación indígena y en el jugo abierto en la toma de decisiones sobre el tipo de oferta escolar (contenidos de aprendizaje y evaluación). Por último, revisa algunas posturas críticas que aducen que el interculturalismo es un movimiento asociado con el neoliberalismo y que para escapar de ese circulo de dominación urgen elaborar un discurso crítico y decolonial. En esa postura crítica se inscriben los movimientos indígenas que luchan no solo por derechos culturales, educativos y lingüísticos, sino contra el capitalismo global.

Palabras clave: Interculturalismo. Educación intercultural. Neoliberalismo. Indigenismo.

\begin{abstract}
The paper reflects on the intercultural educational policies of the last two decades in Mexico. It begins with a presentation of the issues debated in this period and the reflections that intercultural policy has raised. It then analyzes the bureaucracy from the apparatuses that were created in these two decades to promote intercultural policies, which not only sought to dismantle the old apparatus of indigenous integration, but to establish a free market policy, "de bocho y changarro", supported by reforms that triggered changes in the management of resources allocated to indigenous education and in the open juice in decision-making on the type of school offerings (learning content and evaluation). Finally, we review some critical positions that argue that interculturalism is a movement associated with neoliberalism and that in order to break out of this circle of domination it is urgent to develop a critical and decolonial discourse. This critical position includes the indigenous movements that fight not only for cultural, educational and linguistic rights, but also against global capitalism.
\end{abstract}

KeyWords: Interculturalism. Intercultural education. Neoliberalismo. Indigenism.

\section{Introducción}

En las últimas dos décadas, la problemática de la educación intercultural en México como política pública ha sido objeto de intensos debates, reflexiones y hasta de cuestionamientos. En este trabajo nos detendremos en los cuestionamientos que se formulan para las políticas interculturales y algunas reflexiones acerca de las respuestas que surgen en su defensa. Ahora se discute incluso su viabilidad como discurso político, pues parece no satisfacer plenamente a quienes las han promovido (líderes indígenas, 


\section{RevistAleph}

académicos y funcionarios), especialmente de aquellos sectores que se mueven en los círculos académicos y la burocracia de las instituciones de gobierno. Algunas de las críticas dirigen su mirada hacia las políticas interculturales ligadas al neoliberalismo, aduciendo que engendran nuevas formas de discriminación y exclusión.

En este trabajo nos interesa destacar: 1) en primer lugar, la política de "educación intercultural para todos" materializada en el programa de la Coordinación Educación Intercultural Bilingüe (CGIB) y la ampliación de la cobertura de la educación media superior y superior. En tal propósito buscamos articular las ideologías de un complejo campo de debate en el que participan actores (desde funcionarios de gobierno, organizaciones no gubernamentales, hasta organismos internacionales, líderes indígenas, académicos y profesores indígenas) de muy variadas posiciones políticas influenciadas por el discurso intercultural, que van desde la oposición radical a las demandas de reconocimiento de la diversidad cultural -porque suponen aumenta el riesgo a la integridad de la unidad nacional-, hasta posiciones que justifican plenamente las consecuencias del reconocimiento de la pluralidad cultural y la autonomía; 2) en segundo lugar, abordamos los argumentos críticos que sostienen que la interculturalidad funciona como la nueva lógica del capitalismo global, que promueve la interacción de las culturas y enmascara el racismo a través del respeto a la identidad del otro. Es una crítica radical que, sin duda, esconde algunas perversiones y desviaciones asociadas con la ideología pigmentocrática, el criollismo y la supremacía occidental.

En general, las reflexiones tienen la intención de hacer una lectura critica, por un lado, de la promoción de políticas y prácticas inclusivas de las instituciones de gobierno y, por otro, la revisión de los argumentos según los cuales estas políticas sirven a intereses y agendas neoliberales. Sobre todo, nos anima la idea de escribir un par de críticas sobre la interculturalidad como discurso político y discurso pedagógico, ámbitos muy interconectados y que serán discutidos en términos de posibles relaciones connaturales con las políticas neoliberales. 


\section{RevistAleph}

\section{El marco de los debates}

Durante las últimas dos décadas el debate académico sobre la interculturalidad adquirió relevancia como discurso social y político, se acentuó, incluso dentro de nuestros círculos académicos llegó a considerarse como uno de los temas estelares. Ciertamente se realizaron eventos de todo tipo (como foros, conferencias, cursos, seminarios, conversatorios, coloquios, congresos, en los que participamos (en unos casos como promotores y otros como simples observadores), para discutir lo que entendíamos por interculturalidad y por lo que debíamos entender ${ }^{20}$. El número de publicaciones sobre el tema creció significativamente, basta ojear el último volumen sobre El Estado de Conocimiento 2002-2011, coordinado por BERTELY, DIETZ y DIAZ TEPEPA (2013), bajo el título Multiculturalismo y Educación, para tener una idea de la espiral de crecimiento, así como la inusitada expansión del tema hacia otras áreas de conocimiento de las ciencias sociales y humanas.

La producción académica de este periodo tiene un despliegue sin precedentes y el ascenso de un movimiento intelectual que articula desde posiciones críticas hasta posiciones relativistas y fundamentalistas. La mayor parte de tales discusiones estuvieron guiadas por la necesidad de esclarecer lo que significa la interculturalidad en términos conceptuales- y para tener un lenguaje común para quienes participaron en este movimiento desde diferentes frentes. El Documento de la UNESCO de 2007, Directrices sobre educación intercultural sale a la luz precisamente con la intención de ofrecer definiciones, objetivos y un marco operativo que busca reducir tales ambigüedades y clarificar el significado político y pedagógico. En un párrafo inicial del

\footnotetext{
${ }^{20}$ Véase en las referencias bibliográficas una serie de artículos escritos por Rebolledo (2001, 2004, 2005, $2006,2010,2014,2019,2020)$, que dan cuenta de los debates y las tendencias que adopta el discurso intercultural durante este periodo.
} 


\section{RevistAleph}

citado documento podemos encontrar una frase que dice que la "educación intercultural no puede ser un simple añadido al programa de instrucción normal. Debe abarcar el entorno pedagógico como un todo".

Las definiciones conceptuales buscaban entre otras cosas consenso entre los diversos sectores académicos y actores institucionales. En su deseo por manejar conceptos comunes y una jerga institucional compartida sobre la interculturalidad y la diversidad cultural, algunas instituciones de gobierno elaboraron un documento denominado Marco Conceptual de la Campaña Nacional por la Diversidad Cultural de México (EMBRIZ, 2011), cuyo propósito es presentar los "tres conceptos de referencia obligada para la planificación de la acción intercultural" y de ese modo construir un instrumento que ayudará a difundir su significado a todas las comunidades. Se entiende que el documento buscaba definir los conceptos de diversidad cultural e interculturalidad a fin de restar ambigüedades, anteponiéndose a las múltiples definiciones que flotaban en el ambiente, que poco esclarecían el significado "correcto" de los conceptos, y menos contribuían al desarrollo de la política educativa, por el contrario, la tornaban confusa (REBOLLEDO, 2020).

Un segundo componente del discurso intercultural que entró en los debates de las dos últimas décadas está referido a la fuerza que despliega el movimiento indígena, protagonizado por el Ejercito Zapatista de Liberación Nacional (EZLN), la Asamblea Popular de los Pueblos de Oaxaca (APPO) y otras organizaciones de raíz comunitaria. Representa un movimiento indígena en ascenso que contribuyó sin duda a la creación de un nuevo escenario político y un discurso incluyente de interculturalidad. "Un mundo donde quepan todos" no es una simple frase del vocabulario zapatista, pronto se convirtió en un concepto clave que sintetiza muy bien el pensamiento de los pueblos indígenas excluidos (REBOLLEDO, 2003). Pensamos que la filosofía educativa zapatista no solo cuestionó los objetivos de la educación intercultural bilingüe (EIB) que se venía ofreciendo a los pueblos indígenas, sino que plantea la formación de una nación fundada en la pluriculturalidad y multilingüismo, el establecimiento de nuevas formas de 


\section{RevistAleph}

convivencia social, en una nueva educación y una ética basada en el respeto a la dignidad del ser humano. En ese sentido, el reconocimiento de los Acuerdos de San Andrés y la legislación de los derechos y cultura indígenas, es la punta de lanza de la filosofía educativa, el reconocimiento de las autonomías locales y la transformación de las relaciones de los pueblos indígenas con el Estado.

El interculturalismo crítico también se inscribió dentro de debates de las dos últimas décadas, como una corriente de pensamiento que cuestiona el interculturalismo funcional, considerado una ideología que despoja el carácter político de las demandas indígenas y promueve el relativismo cultural. El interculturalismo crítico es un movimiento intelectual que instiga a reconocer que la diferencia cultural se construye a partir de la una matriz colonial de poder racializado y jerarquizado (WALSH, 2008). Como proyecto intelectual de emancipación epistémica induce a explorar sobre nuevos horizontes de producción de conocimientos y sobre la coexistencia de diferentes formas de conocimiento, tanto de aquellos que son producidos por la academia como los que emergen de la experiencia de los movimientos sociales. La colonialidad del poder, del saber y del ser, son conceptos claves para este proyecto intelectual, cuya estrategia va más allá de la descolonización, supone la reconstrucción radical del poder, del saber y del ser.

\section{Los aparatos de la política intercultural}

La política de educación intercultural crea aparatos institucionales con los cuales expande políticas de la diversidad y opera los diferentes programas. Antes de pasar a exponer el tema en cuestión, vale la pena hablar un poco sobre los aparatos institucionales y el poder que ejercen. Según Weber (1987, p. 175) "el tipo más puro de dominación legal es aquel que se ejerce por medio de un cuadro administrativo burocrático". El centralismo y la función pública es ejercida como un cargo burocrático sometido a rigurosa vigilancia administrativa (ahora la función pública pasa por la 


\section{RevistAleph}

lectura estricta de los manuales de operación y la rendición de cuentas). Los programas de educación intercultural y otros ligados a éstos no escapan a los controles burocráticos, no están exentos de la aplicación de los instrumentos de supervisión administrativa y del saber profesional especializado. Siguiendo a Weber, estos aparatos que operan la política intercultural tiene el control institucional gracias a un saber profesional con el cual se nutre su poder racional y un saber en el servicio público que otorga a los funcionarios facultades profesionales para ejecutarlos.

Las instituciones que nacieron para impulsar las políticas de la diversidad y la educación intercultural para todos, en orden de fundación, son: la desaparición en el año 2000 del Instituto Nacional Indigenista (INI) y la creación de un nuevo aparato encargado de la política indigenista, la Coordinación para el Desarrollo Integral de los Pueblos Indígenas (CDI), en 2001 se funda la Coordinación General de Educación Intercultural Bilingüe (CGEIB), en 2003 el Instituto Nacional de las Lenguas Indígenas (INALI) y el sistema de Universidades Interculturales (UI). Son tres las instituciones, además de las 11 UI que se crean para encauzar la nueva política indigenista de los gobiernos neoliberales, fundamentada en la diversidad cultural y lingüística. Cabe mencionar que la Dirección General de Educación Indígena (DGEI) fundada en 1978, se mantiene como institución responsable del subsistema de educación básica, se encarga desde entonces hasta ahora, de trazar las políticas de educación indígena, la actualización docente y la elaboración y diseño de los materiales didácticos. Cabe decir que la CDI fue fundada sobre las ruinas de la estructura del INI y el indigenismo como política fue sustituido por una nueva jerga empresarial neoindigenista de dotación de "bocho y changarro", por tanto, en sentido estricto no se creo una nueva institución, sino una nueva política indigenista de emprendedores. Al final fueron únicamente dos instituciones nuevas las que se crearon y fueron a través de éstas que se sentaron las bases de una política intercultural y bilingüe, a las que se suma la creación de las UI.

Este cambio dio lugar a reacomodos en las burocracias encargadas de las políticas públicas y sobre todo produjo nuevos discursos que vendrían a legitimar a los 


\section{RevistAleph}

gobiernos en turno. La desaparición de unas instituciones y la fundación de otras fue la característica de este periodo, sobre todo el surgimiento de un discurso intercultural que buscaba entre otras cosas dar respuesta a la rebelión zapatista de 1994 y a los Acuerdos de San Andrés Larrainzar de 1996. El Plan Nacional de Desarrollo Educativo (PNDE 2001-2006) fue una de las primeras respuestas que representan las líneas generales de la política intercultural y que a todas luces personifica la moneda de cambio para este nuevo gobierno para el movimiento indígena: políticas de interculturalidad y derechos culturales y lingüísticos individuales por derechos colectivos, educación intercultural pública por educación autónoma, derechos lingüísticos plenos por protección a la discriminación lingüística.

En resumen, la política de educación intercultural para todos, planeta la creación de un sistema educativo integral cuyo eje principal es la promoción de la interculturalidad a través de acciones trasversales de gobierno, con el principio de que no se trata de promover la educación intercultural solo para indígenas, sino para toda la población. La interculturalidad definida como la interacción entre culturas diferentes, la convivencia armónica de personas con culturas diferentes, el diálogo cultural, el reconocimiento y el respeto a las particularidades de cada una de las culturas en contacto. La instauración de un sistema de derechos fundamentales donde cada persona podrá ser educado de acuerdo con su propia cultura e idioma y orientado a proteger y fortalecer las lenguas indígenas.

La creación de las $\mathrm{UI}^{21}$, en sus objetivos reiteran la importancia de atender a los

\footnotetext{
${ }^{21}$ Hay 11 universidades interculturales en funcionamiento. Ellas están situadas en regiones indígenas de los estados de e México, Chiapas, Tabasco, Veracruz, Puebla, Sinaloa, Michoacán, Quintana Roo, Guerrero, Nayarit e San Luis Potosí. En ciclo escolar 2010-2011, las universidades contaban con 6.897 alumnos, de los cuales 3.713 eran mujeres (54\%), y 3.184, hombres (46\%). Más de la mitad (53\%) de todos los alumnos matriculados eran jóvenes que aun hablaban sus idiomas nativos, o sea 3.670 alumnos (1.926 mujeres y 1.744 hombres. Más de 40 lenguas indígenas están presentes en estas universidades (SALMERON, 2018, p. 100).
} 


\section{RevistAleph}

jóvenes indígenas, ofreciendo alternativas de formación profesional para el desarrollo social y económico de sus respectivas regiones, arraigarlos en sus lugares de origen y ofrecerles oportunidades focalizando sus lenguas, culturas y saberes locales como materia de las políticas curriculares. Desde que se fundan hasta la actualidad, estos y otros temas ocupan progresivamente el centro de las reflexiones. La política intercultural y los espacios públicos (municipales y comunales) plantean que las UI están pensadas para sustentar sus programas en la formación profesional, la investigación anclada a las problemáticas locales, la vinculación con las comunidades, la cooperación académica nacional y la internacionalización. Algunos programas reportan resultados positivos respecto a la vinculación con las comunidades con las que mantienen relación activa (DIETZ, 2018) y otros no han logrado romper con el academicismo que las enclaustra y los aísla de las comunidades. Una característica que define a las UI es el pobre financiamiento con el que operan regularmente, año con año esperan mejorar incrementando sus recursos para la investigación y la vinculación, sin embargo, parece que es una tendencia que no logran revertir. En vez de mejorar, empeoran en términos de crear especialidades y nuevas ofertas vinculadas a las comunidades. Estudios longitudinales elaborados por investigadores dedicados a focalizar los problemas de la educación de los jóvenes indígenas que se forman en las UI (PÉREZ, M. L., RUIZ, V y VELASCO, S., 2015), observan que los estudiantes después de que egresan tienen dificultades de conseguir empleo para lo que fueron formados y muchos se emplean en otros oficios, creando así una imagen negativa de las profesiones que ofertan las UI y produce en consecuencia un fenómeno de empobrecimiento intelectual que va siendo propio de estas instituciones enclavadas en las regiones indígenas.

Hoy las UI ofertan programas, que a la luz de los diagnósticos enfrentan varios desafíos: una formación profesional cada vez más exigente a nivel local y global, cada vez más creciente y expansiva en cuanto a la cobertura escolar, cada vez más compleja en organización del conocimiento de los currículos (dialogo de saberes) y los métodos de enseñanza (conflictos inter epistémicos). Comentaremos brevemente en seguida 


\section{RevistAleph}

sobre las dos cuestiones fundamentales. En primer lugar, ofrece formación de profesional en nuevos campos de conocimiento (desarrollo regional, derecho, cultura y lengua indígena, medicina tradicional, turismo, entre otras), ligados a demandas del contexto local, que pasa por el filtro de las políticas interculturales (planes curriculares diferenciados y específicos), pero también por el tamiz de los organismos internacionales y las presiones del mercado global (evaluaciones estandarizadas).

Y, en segundo lugar, el impacto de la globalización sobre las culturas indígenas es muy notable (se expresa en el saqueo, el despojo, la depredación del medio ambiente, el desplazamiento lingüístico y el etnocidio) y por eso las UI están teniendo un papel importante en la creación de perspectivas que buscan revertir esa situación a nivel local. No solo están creando opciones de empleo y desarrollo local, sino que están sirviendo como muros de contención a la invasión cultural y lingüística, la emigración y la pobreza.

\section{Interculturalismo y neoliberalismo}

Algunos estudiosos del interculturalismo sostienen que el interculturalismo y el neoliberalismo se asocian en algún punto en el curso de la política (ŽlŽEK, 1998), aún cuando surgen como movimientos históricos antagónicos. En los hechos compartan una agenda común que se enmarca en la lucha por los derechos humanos educativos, culturales y lingüísticos. Mientras el interculturalismo es un movimiento encabezado por minorías culturales desposeídas, el neoliberalismo es un movimiento económico del capitalismo global. El neoliberalismo entra en escena para dar un carácter distinto a las luchas de las minorías culturales, es decir, implanta una lógica implacable de deslegitimación de las luchas de estas mismas minorías. Es aquí donde convergen el interculturalismo y la doctrina económica neoliberal, es en esta zona del discurso en la que se enmarcan las respuestas que el estado elabora para frenar los movimientos 


\section{RevistAleph}

indígenas. El diálogo intercultural en este caso representa una acción justificada del estado para frenar la exigencia autonómica de los movimientos indígenas.

Nos parece importante distinguir el interculturalismo del neoliberalismo, tanto a nivel discursivo como político, porque se difunde en la actualidad una crítica radical que asocia la interculturalidad con la ideología del capitalismo global, arguyendo que cuando logra tener conexión con las culturas locales, las vacía, las deja sin contenido, las convierte en objetos chatarra y las arroja a los basureros de las culturas, se comporta como empresa extractivista y depredadora. En otros casos, traza distancias con las culturas locales o no se enraíza en ninguna parte y mantiene una distancia gracias a su posición universalista. El respeto multiculturalista por la cultura del otro es precisamente la forma en que se reafirma la superioridad y el privilegio eurocentrista. El respeto que proclama es:

[...] un racismo con distancia: respeta la identidad del Otro, concibiendo a éste como una comunidad "auténtica" cerrada, hacia la cual él, el multiculturalista, mantiene una distancia que se hace posible gracias a su posición universal privilegiada" (ŽIŽEK, 1998, p. 172).

Antes de examinar las ideologías neoliberales que están detrás de las prácticas interculturales, es importante identificar los movimientos discursivos y los debates académicos que nutren la idea de una relación estrecha entre interculturalidad y neoliberalismo. La ecuación interculturalismo es igual al neoliberalismo es una operación que engloba varias cuestiones a discutir. Identificamos, por ejemplo, un rasgo fundamental del interculturalismo asociado con el neoliberalismo: el discurso intercultural como política de las minorías adquiere relevancia justo en el momento en que los discursos neoliberales también se vuelven dominantes. Eso no significa que debamos entender que las luchas de las minorías se acompasan con el orden capitalista, por el contrario, ante todo son movimientos anticapitalistas que buscan distanciarse y oponerse a él. Por lo tanto, no hay que adjudicar a los interculturalistas los males causados por las perversidades neoliberales. Esta idea puede ayudarnos a entender que 


\section{RevistAleph}

los movimientos sociales convergentes con los programas neoliberales no tienen que indicar necesariamente una relación causa-efecto. Las políticas multiculturales que engloban derechos de autogobierno y exigencias legales sobre uso de las lenguas maternas son formas de derechos de las minorías que limitan o modifican su relación con los estados nación (KYMLICKA, 2003). Algunos estudiosos críticos marcan diferencias entre interculturalismo funcional auspiciado por el estado neoliberal y el interculturalismo crítico decolonial de las organizaciones indígenas (WALSH, 2008 y MIGNOLO, 2016).

Un concepto que puede ayudarnos a entender la compleja relación entre el interculturalismo y el neoliberalismo, es el de hibridación. El concepto de hibridación de las prácticas interculturales es utilizado por las agencias neoliberales y los programas educativos de los gobiernos, incluso los propios proyectos interculturales de las organizaciones indígena suelen identificarse con tales prácticas de hibridación cuando crean pequeñas empresas cooperativas. Hay que señalar, sin embargo, que los interculturalistas críticos poscoloniales raramente expresan sus posiciones teóricas en términos de hibridez de contrarios, sino de luchas de contrarios. A este respecto Bhabha (1999) hace una distinción entre hibridez como producto frente a la hibridez como práctica:

[...] la hibridación es realmente acerca de cómo se negocia entre textos o culturas o prácticas en una situación de desequilibrios de poder, para poder ver la manera en que las estrategias de apropiación, revisión e interacción pueden producir posibilidades para los menos favorecidos, para poder captar en un momento de emergencia, en el proceso mismo del intercambio o la negociación, la ventaja. [ . . .] la hibridación es un proceso discursivo, enunciatorio, cultural y subjetivo que tiene que ver con la lucha en torno a la autoridad, la autorización, la desautorización y la revisión de la autoridad (BHABHA, 1999, p. 64).

Desde esta perspectiva, la hibridación como noción es diferente a la hibridación como práctica. Normalmente las críticas se dirigen a la definición de hibridación como atributo personal escencializado, como posición del sujeto individual y no como 


\section{RevistAleph}

identidad de grupo. En educación intercultural es innegable la posición de que la hibridación parte del sujeto individual que aprende y que aprende una cultura escolar no hibrida. En otros casos la hibridación se relaciona con la comunicación escolar y donde la comunicación pedagógica es favorecida por la hibridación y la mezcla de estilos comunicativos esenciales en la cultura académica es la clave para el aprendizaje escolar en situaciones multilingües. Los interculturalistas críticos analizan cómo las lenguas en conflicto, los valores o las identidades culturales, son negociadas con las normas dominantes de la lengua vehicular del curriculum escolar. En estos contextos la voz de los estudiantes minoritarios, la lengua materna y la representación cultural, juegan un papel importante en las situaciones de negociación. Siguiendo a Bhabha (1999), la hibridación es una forma de negociación estratégica para resistir a valores y normas dominantes que podrían silenciar o privar a las personas de representarse a si mismos en su propia cultura.

Las críticas focalizan su mirada en las políticas interculturales ligadas al neoliberalismo, porque aducen que las políticas públicas de los gobiernos neoliberales no han sabido afrontar las tendencias amenazantes de la cultura global y la matriz racista del sistema educativo, dan a entender que el interculturalismo no sido capaz de ofrecer salidas y piensan además que éstas deberían tener una llave secreta de la puerta de salida. Lo cierto es que la interculturalidad se ha tornado en un poderoso discurso político que ha ganado terreno en las instituciones de gobierno, muy ligado con los movimientos sociales indígenas, incluso dentro de la propia academia se ha posesionado como un importante objeto investigación, en un campo de análisis político y de acción educativa, pero también ha contribuido a la formación de un campo de batalla para quienes se disputan el control de los recursos culturales.

No obstante que el tema de la educación de los pueblos indígenas está enunciado dentro de los programas de gobierno, éste aparece alojado en uno de los capítulos de la "educación intercultural para todos" y subsumido a su vez a los aspectos generales de las acciones que los gobiernos neoliberales desplegaron durante las dos 


\section{RevistAleph}

últimas décadas, que traducidas en términos de presupuesto destinado para la educación intercultural indígena, se reduce a poco menos que nada, comparado con los recursos destinados a promover el discurso de la interculturalidad para todos. Por otra parte, se advertía de que este objetivo ha dado lugar a cambios superficiales en la educación indígena, argumentando que las políticas interculturales fueron diseñadas por las elites neoliberales y reforzadas por alianzas tácticas con partidos políticos, con la clara intención de desviar la atención de los movimientos indígenas (REBOLLEDO, 2004 y 2005).

En los últimos tiempos ha entrado a escena el interculturalismo crítico decolonial con influencia intelectual en reducidos grupos académicos, que reconoce que la diferencia cultural se construye a partir de la una matriz colonial de poder racializado y jerarquizado (WALSH, 2006). Es un proyecto intelectual de emancipación epistémica que explora sobre nuevos horizontes de producción de conocimientos y sobre la coexistencia de diferentes formas de conocimiento, tanto de aquellos que son producidos por la academia como los que emergen de la experiencia de los movimientos de sociales. La colonialidad del poder, del saber y ser son conceptos claves para este proyecto intelectual, cuya estrategia va más allá de la descolonización, supone la reconstrucción radical del poder, del saber y del ser.

\section{Conclusiones}

Aun no contamos con suficientes elementos cuantitativos ni cualitativos que permitan hacer un balance riguroso sobre el impacto de dichas políticas, sin embargo, como vimos el debate político y académico actual nos proporciona un amplio abanico de reflexiones sobre el estado de la cuestión. El interculturalismo de los gobiernos neoliberales ha tenido matices, proponen la integración de la población indígena al mercado (y no a la nación mexicana como con el indigenismo) y en ese sentido el subsistema de educación indígena adopta ese enfoque. 


\section{RevistAleph}

Por último, mientras algunas instituciones promueven el interculturalismo y el multilingüismo, otras promueven la eficacia y la obtención de ganancias aplicando la misma política con los programas educativos (una lengua vehicular del curriculum y una cultura escolar común, universal). Así de contradictorias son las políticas públicas de los gobiernos neoliberales. Las nociones de productividad y especialización siguen dominando el discurso educativo, convirtiendo la comunicación pedagógica en un instrumento de eficiencia. No obstante que los programas educativos interculturales de los gobiernos neoliberales reconocen el valor económico y simbólico de las culturas y lenguas indígenas, en el contexto de las escuelas y de la enseñanza, desdeñan el multilingüismo y el multiculturalismo como materia de trabajo escolar, más bien los destinan a ser parte de la interacción en los contextos informales de comunicación y esperan que desde ahí se desplieguen con naturalidad.

\section{Referências}

BERTELY, María., DIETZ, Gunther y DIAZ, Guadalupe (Coordinadores). Multiculturalismo y educación, 2002-2011, Estados del Conocimiento, México: Asociación Nacional de Universidades e Instituciones de Educación Superior/Consejo Mexicano de Investigación Educativa, 2013.

BHABHA, Homi. El lugar de la cultura. Buenos Aires: Ediciones Manantial, 1999.

KYMLICKA, Will. La política vernácula. Nacionalismo, multiculturalismo y ciudadanía. Barcelona: Editorial Paidós, 2003.

MIGNOLO, Walter. Hacer, pensar y vivir la modernidad. México: Editorial Borde Sur, 2016.

PEREZ, Maya Lorena, RUIZ, Verónica y VELASCO, Saúl. Interculturalidad(es). Jóvenes indígenas: educación y migración. México: Colección Horizontes Educativos, Universidad Pedagógica Nacional, 2015.

REBOLLEDO, Nicanor. Educación indígena y neozapatismo. En: Anuario Educativo Mexicano, Visión Retrospectiva, Guadelupe Teresinha Bertussi, Coordinadora. México: Ediciones La Jornada/UPN, 2001. 


\section{RevistAleph}

REBOLLEDO, Nicanor. Interculturalismo y autonomía. Reflexiones en torno al movimiento indígena y las políticas educativas. En: Anuario Educativo Mexicano. Visión Retrospectiva. México: Universidad Pedagógica Nacional /Porrúa Hermanos Editores, 2004.

REBOLLEDO, Nicanor. Interculturalismo y autonomía: las universidades indígenas y las políticas de alteridad. En: NAVARRO, Cesar (Coordinador). La mala educación en tiempos de la derecha. México: Universidad Pedagógica Nacional/Porrua Hermanos Editores, 2005.

REBOLLEDO, Nicanor. Educación comunitaria y comunalismo rural. Reflexiones en torno a experiencias de bachillerato indígena. En: ROSAS, Lesvia (Coordinadora). La Educación Rural en México en el Siglo XXI. México: Ediciones Centro de Estudios Educativos/CREFAL, pp. 509$5442,2006$.

RICO, Sergio y REBOLLEDO, Nicanor. Educación y comunidad. Prácticas autonómicas en la Mazateca Alta. México: Universidad Pedagógica Nacional, Colección Polvo de Gis, 2010.

REBOLLEDO, Nicanor. Universidade e diversidade. Reflexões antropológicas sobre um caso paradigmático de formação de professores indígenas bilíngues no México. En: TASSINARI, Antonella., DE ALMEIDA, Nilton e REBOLLEDO, Nicanor (Organização). Diversidade, educação e infância. Reflexões antropológicas. Brasil: Editora UFSC, pp. 343-369, 2014.

REBOLLEDO, Nicanor. Antropología de una profesión indígena en México. En: TASSINARI, Antonella, OLIVEIRA, Deise Lecy e VIERIA, José (Organizadores). Antropologia e educação. Refletindo sobre processos educativos em contextos escolares, não escolares e de políticas públicas. Brasil: Editora Copiart, Editora da Universidade Federal do Amazonas e Editora da Universidade Federal do Rio Grande do Norte, pp. 157-177, 2019.

REBOLLEDO, Nicanor. La interculturalidad en las aulas. En: Interculturalidad y diversidad en la educación: concepciones, políticas y prácticas. Sonia Comboni Salinas José Manuel Juárez Núñez (Editores). México: Universidad Autónoma Metropolitana-Xochimilco, pp. 163-180, 2020.

SALMERON, Fernando. O programa Universidades Interculturais no México: principais problemas e perspectivas de uma experiência prática. En: SOUZA, Antonio Carlos, DOS SANTOS, Luis Felipe e LINS, Gustavo (Organizadores). Interculturalidade(s). Entre ideias, retóricas e práticas em cinco países da América Latina. Brasil: Universidade Federal do Rio de Janeiro, pp. 97-120, 2018.

WALSH, Catherine. Interculturalidad, plurinacionalidad y decolonialidad:

las insurgencias político-epistémicas de refundar el Estado. En: Revista Tabula Rasa, No.9: 131-152, julio-diciembre, pp. 134-152, 2008.

WEBER, Max. Economía y sociedad. México: Fondo de Cultura Económica, 1984. 


\section{RevistAleph}

ŽIŽEK, Saljov. Multiculturalismo, o la lógica cultural del capitalismo multinacional. En: FREDRIC, Jameson y ŽlŽEK, Slajov. Estudios culturales. Reflexiones sobre el multiculturalismo.

Barcelona: Editorial Paidós, pp.137-188, 1998.

Data do envio: $24 / 06 / 2020$

Data do aceite: $13 / 04 / 2021$ 